\title{
Comparison of High- and Low-Energy Collision-Induced Dissociation Tandem Mass Spectrometry in the Analysis of Glycoalkaloids and their Aglycons
}

\author{
Magda Claeys and Hilde Van den Heuvel \\ Department of Pharmaceutical Sciences, University of Antwerp, B-2610, Antwerpen (Wilrijk), Belgium \\ Su Chen and Peter J. Derrick \\ Warwick Institute of Mass Spectrometry, University of Warwick, Coventry CV4 7AL, United Kingdom
}

Fred A. Mellon and Keith R. Price

Institute of Food Research, Norwich Laboratory, Norwich Research Park, Colney, Norwich NR4 7UA, United Kingdom

Four aglycons (tomatidine, demissidine, solanidine, and solasodine) and three glycoalkaloids ( $\alpha$-tomatine, $\alpha$-chaconine, and $\alpha$-solanine) have been analyzed by positive ion liquid secondary ion high-energy and low-energy collision-induced dissociation (CID) tandem mass spectrometry, performed on a four-sector (EBEB) and a hybrid (EBQQ) instrument, respectively. Both high- and low-energy collision-induced dissociation mass spectra of $[\mathrm{M}+\mathrm{H}]^{+}$ ions of these compounds provided structural information that aided the characterization of the different aglycons and of the carbohydrate sequence and linkage sites in the glycoalkaloids. Low-energy CID favors charge-driven fragmentation of the aglycon rings, whilst high-energy CID spectra are more complex and contain additional ions that appear to result from charge-remote fragmentations, multiple cleavages, or complex charge-driven rearrangements. With respect to the structural characterization of the carbohydrate part, low-energy CID fragmentations of sugar residues in the glycoalkaloids generate $Y_{n}^{+}$ions and some low intensity $Z_{n}^{+}$ions; the high-energy spectra also exhibit strong ${ }^{1,5} \mathrm{X}_{n}^{+}$ions, formed by multiple cleavage of the sugar ring, and significant $Z_{n}^{+}$ions. (J Am Soc Mass Spectrom 1996, 7, 173-181)

$\mathrm{M}$ ass spectrometry is playing an increasingly important role in the structural analysis of complex biomolecules. Tandem mass spectrometry has demonstrable advantages over conventional mass spectrometry in the structural analysis of saponins and related compounds [1-3]. The fragmentation modes of a range of glycoalkaloids, isolated from potato shoots and green tomatoes, have been determined recently by positive and negative liquid secondary ion four-sector tandem mass spectrometry with scanning-array detection [4, 5]. Considerable structural information, which includes the nature of various aglycons and the linkage and position of sugars in the glycoalkaloids was elucidated from the tandem mass spectra of the $[\mathrm{M}+\mathrm{H}]^{+}$and $[\mathrm{M}-\mathrm{H}]^{-}$ions of these compounds.

Most reported collision-induced dissociation (CID) studies of steroid derivatives have concentrated on bile

Address reprint requests to Professor Magda Claeys, Departement Farmaceutische Wetenschappen, Universitaire Instelling Antwerpen, Universiteitsplein 1, B-2610, Antwerpen (Wilrijk), Belgium. salts and steroid conjugates (sulfates and glucuronides) and have been conducted on $[\mathrm{M}+\mathrm{Na}]^{+}$ and $[\mathrm{M}-\mathrm{H}]^{-}$ions, which contain a stable charge site. The successful application of high-energy CID to the structural analysis of bile acids first was described by Liehr et al. [6]. These authors examined the $[\mathrm{M}+\mathrm{Na}]^{+}$ ions of isomeric bile salts, which had a polar side chain that contained taurine or glycine at $\mathrm{C} 17$, by CID/mass-analyzed ion kinetic energy spectroscopy and recorded structurally informative charge-remote fragmentations in the A, B, C, and D rings. Tomer and Gross [7] studied a series of steroid conjugates (sulfates and glucuronides) and bile salts and concluded that CID of the $[\mathrm{M}-\mathrm{H}]^{-}$ions of steroid glucuronides resulted in charge-driven reactions, whereas CID of the $[\mathrm{M}-\mathrm{H}]^{-}$ions of sulfates and bile salts generated charge-remote fragmentations. Griffiths et al. [8, 9] examined the charge-remote fragmentation patterns of $[\mathrm{M}-\mathrm{H}]^{-}$ions of sulfated and glucuronidated bile acids and their amino-sulfonic acid derivatives in detail and showed that taurine-conjugated bile acids yielded more intense and informative CID spectra than 
unconjugated bile acids and that 3-, 7-, and 12sulfonylcholate isomers could be differentiated by recording the fast-atom bombardment-collision-induced dissociation (FAB/CID) spectra of their derivatized and underivatized forms. Low-energy CID spectra of $[\mathrm{M}-\mathrm{H}]^{-}$ions of bile salts, which were formed by fast-atom bombardment (FAB) or thermospray ionization, were reported by Libert et al. [10] and Eckers et al. [11], respectively. It is interesting to note that fragmentation reactions that yield abundant product ions are found to occur in the $B$ ring in the case of unsaturation at $\mathrm{C} 4$ or the presence of a hydroxyl group at C7. Product ions with low abundances, which correspond to fragmentation in the $C$ and $D$ rings, also are present in the low-energy CID spectra of FAB-generated $[\mathrm{M}-\mathrm{H}]^{-}$ions [10]. Wood et al. [12] recorded B/E linked scan spectra of the unimolecular decompositions of $[\mathrm{M}+\mathrm{Na}]^{+}$ions of glycine- and taurine-conjugated bile acids and reported B- and C-ring cleavages if a hydroxyl group was present at C7 or C8 and $\mathrm{C} 12$, respectively. In a very recent study, de Hoffmann and co-workers [13] examined the low-energy CID fragmentation of tauro-conjugated bile acid anions in detail and established that the low-energy dissociations result from two general mechanisms: chargedriven and charge-remote fragmentations. Chargedriven fragmentations were found to dominate the spectra of saturated ring compounds. These reactions resulted in the loss of parts of the side chain as neutral species and yielded useful information on structural isomers. Charge-remote fragmentations were found to be more intense in the presence of unsaturation and also provided information on the positions of unsaturation or of hydroxy or keto groups in the rings. Tandem mass spectrometry of protonated molecules formed by positive chemical ionization of anabolic steroids yields ring $B$ fragmentations of sufficient intensity to be useful in the analysis of these molecules in biological fluids [14]. A combination of high-energy collision-induced dissociation (CID) tandem mass spectrometry and ${ }^{13} \mathrm{C}$-labeling recently has been used to determine the positions of conjugation of bile acids with glucose and $N$-acetylglucosamine [15]. The lowenergy CID and unimolecular decomposition studies discussed in the preceding text indicate that high-energy collisional activation is not absolutely necessary for the formation of structurally informative fragment ions from the steroid nucleus.

In this study we have compared low- and highenergy CID spectra of the $[\mathrm{M}+\mathrm{H}]^{+}$ions of the $\mathrm{N}$-containing steroids tomatidine, solasodine, demissidine, and solanidine and of the glycoalkaloids $\alpha$ tomatine, $\alpha$-chaconine, and $\alpha$-solanine to determine to what extent low- and high-energy CID can provide information on the structures of aglycon and carbohydrate moieties. The low-energy CID spectra also were inspected in an effort to define which particular fragmentations, among the many typically observed dur- ing high-energy CID, also can be induced by lowenergy collisions.

\section{Experimental}

\section{Materials}

Aglycon standards, which include tomatidine, demissidine, solanidine, and solasodine, and $\alpha$-tomatine were obtained from Sigma Chemical Co. (Poole, Dorset, UK). $\alpha$-Chaconine and $\alpha$-solanine were purified from a crude sample by chromatography as described previously [16].

\section{Mass Spectrometry}

Low-energy CID spectra of $[\mathrm{M}+\mathrm{H}]^{+}$ions of the samples were obtained on a VG70SEQ hybrid instrument (Fisons Analytical, Manchester, UK). Cesium ions with a translational energy of $18 \mathrm{keV}$ and a beam flux of 0.3 $\mu \mathrm{A}$ were used as the ionization beam. The accelerating voltage in the source was $8 \mathrm{kV}$. Precursor ions were mass-selected with a resolution of approximately 1000 by using the double-focusing electric-magnetic sector (EB) part of the instrument. CID was performed in the quadrupole collision cell with argon as collision gas at a pressure of approximately $5 \times 10^{-6}$ mbar (measured in the quadrupole housing) and at a collision energy of $50 \mathrm{eV}$ or lower. The instrument was tuned for maximum transmission of the precursor ions. The product ion spectra were obtained in the continuum acquisition mode with accumulation of 10-20 scans at unit resolution of the quadrupole mass analyzer.

High-energy $\mathrm{CID}$ of $[\mathrm{M}+\mathrm{H}]^{+}$ions was performed on a CONCEPT II $\mathrm{HH}$ four-sector mass spectrometer (Kratos Analytical, Manchester, UK), equipped with a liquid secondary ion source and a scanning-array detection system [5]. The translational energy of the cesium ions was $15 \mathrm{keV}$. The mass spectrometer was operated at 8-kV accelerating voltage. All parameters were controlled by a Kratos MACH 3 system. Tandem mass spectrometry was conducted by using the first two sectors (E1B1), which comprise MS-1, to select a precursor ion and the second two sectors $\left(E_{2} B_{2}\right)$, which comprise MS-2, to analyze the product ions. Precursor ions were mass-selected at a resolution of 1000 to separate the ${ }^{12} \mathrm{C}$ peak in the isotope cluster of the precursor ions. CID of the precursor ions was carried out in a flexicell, which was floated at a potential of 4 $\mathrm{kV}$ relative to Earth. Helium (Air Products, East Rutherford, NJ) was used as the collision gas at a pressure sufficient to attenuate the precursor ion beam by approximately 70\%. 3-Nitrobenzyl alcohol was selected as the liquid matrix because intense $[\mathrm{M}+\mathrm{H}]^{+}$ signals were obtained for both the aglycons and the glycoalkaloids. The product ion spectra were obtained with 1-2- $\mu$ g sample quantities. 


\section{Results and Discussion}

The low- and high-energy CID spectra of the $[\mathrm{M}+\mathrm{H}]^{+}$ ions of the aglycons tomatidine, solasodine, demissidine, and solanidine and the glycoalkaloids $\alpha$-tomatine, $\alpha$-chaconine, and $\alpha$-solanine are shown in Figures 1-7 respectively. The fragmentation reactions of these molecules are discussed individually in subsequent text. Low-energy CID spectra were obtained using argon as the collision gas at an energy of $50 \mathrm{eV}$ (laboratory frame) or lower, whilst high-energy CID spectra were recorded with helium at an energy of $4 \mathrm{keV}$ (laboratory frame), conditions which correspond to maximum energies ( $E_{\mathrm{cm}}$ values) of $2-4.5$ and $16-40 \mathrm{eV}$ for low- and high-energy CID, respectively.

\section{Tomatidine}

Fragmentations observed in the low-energy CID spectrum of tomatidine $[\mathrm{M}+\mathrm{H}]^{+}$ions (Figure 1a) are summarized in Scheme I. The ions at $\mathrm{m} / \mathrm{z} 70$ (not recorded in the high-energy spectrum) correspond formally to $\left[\mathrm{C}_{4} \mathrm{H}_{8} \mathrm{~N}\right]^{+}$and result from fragmentation in the N-containing ring. The ions at $\mathrm{m} / z 114$ and $126 / 124$ can be rationalized by invoking multiple cleavages in the $\mathrm{E}$ ring. Note that ions at $\mathrm{m} / \mathrm{z} 114$ also are formed during electron ionization (EI): Budzikiewicz [17] reported an EI spectrum of solasodine, a structurally

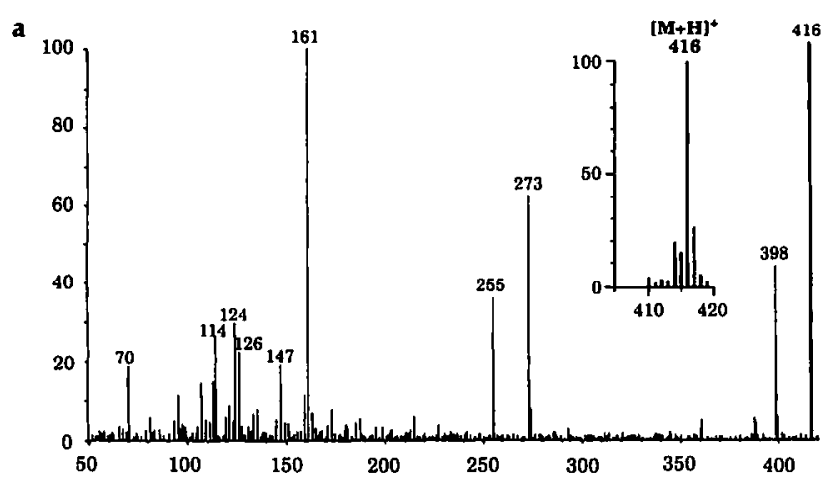

b

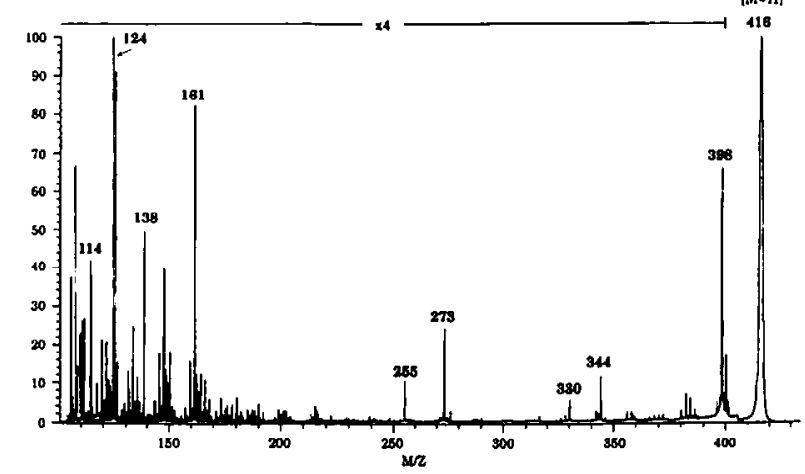

Figure 1. (a) Low-energy and (b) High-energy CID spectra of protonated molecules of tomatidine. The molecule ion region of the (first-order) FAB spectrum (measured with the hybrid instrument) is shown in the insert of the low-energy CID spectrum (a).

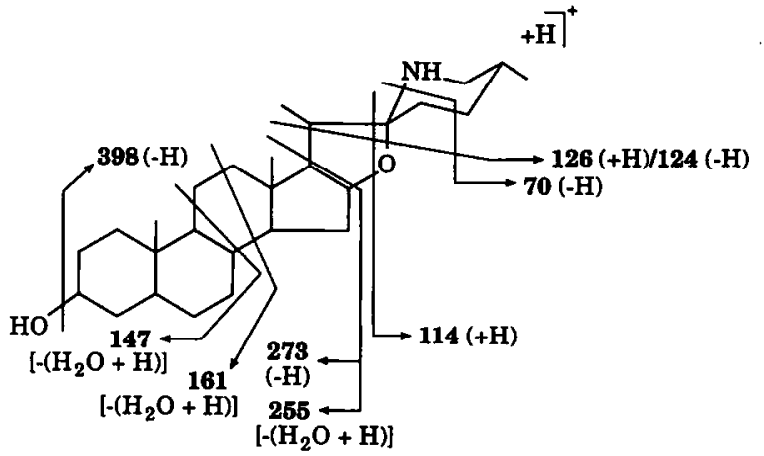

Scheme I. Low-energy CID fragmentations of tomatidine.

related alkaloid that contains unsaturation at C5 (see following text), in which the base peak was at $m / z$ 114. The ions at $m / z 273$ can be interpreted as arising from E-ring fragmentation; a plausible pathway that gives rise to resonance-stabilized allylic ions is shown in Scheme II. The ions at $m / z 255$ may be formed by loss of water from the $\mathrm{m} / \mathrm{z} 273$ ions, as well as by fragmentation of $\left[\mathrm{M}+\mathrm{H}-\mathrm{H}_{2} \mathrm{O}\right]^{+}$ions $(\mathrm{m} / z$ 398). A product ion spectrum (not shown) obtained by selection of $\mathrm{m} / \mathrm{z} 398$ ions as precursor indeed showed that $\mathrm{m} / \mathrm{z} 255$ can be formed directly from $\mathrm{m} / \mathrm{z} 398$. The ions at $\mathrm{m} / z 161$ and 147 can be rationalized as the result of water loss and multiple cleavages in the $C$ ring. A plausible charge-driven mechanism, which involves the generation of resonance-stabilized allylic ions, is suggested in Scheme II.

The high-energy CID fragmentations observed with the four-sector instrument (Figure 1b) are summarized in Scheme III. The ions at $m / z 344,330$, and 138 appear to be characteristic of high-energy CID processes, because they are absent from the low-energy spectrum. The ions at $\mathrm{m} / \mathrm{z} 344$ can be interpreted in a straightforward manner as the result of charge-remote fragmentation in the A ring. The ions at $\mathrm{m} / \mathrm{z} 330$ and 138 are more difficult to rationalize and may involve multiple cleavages in neighboring rings and complex rearrangements at $C 5$ and $C 17$, respectively. It is notable that the ions at $\mathrm{m} / \mathrm{z} 330$ could not be detected in
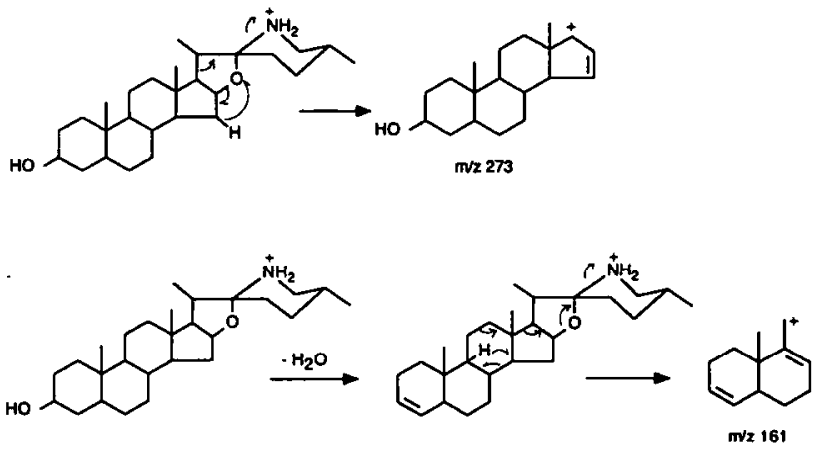

Scheme II. Mechanisms that rationalize the formation of $m / z$ 273 and 161 in the CID spectrum of tomatidine. 


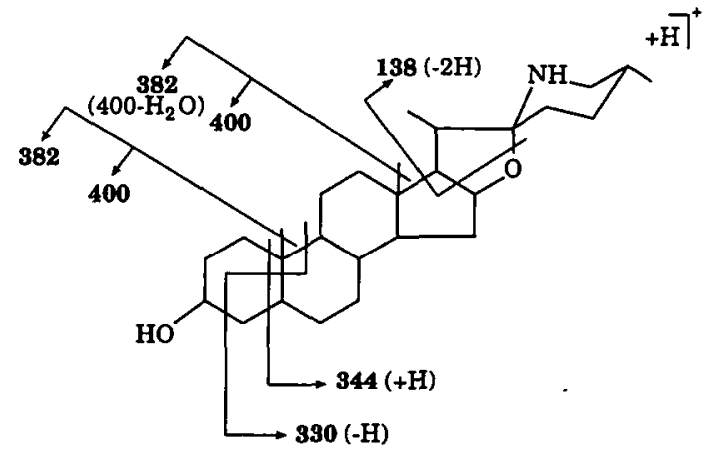

Scheme III. High-energy CID fragmentations of tomatidine.

a CID spectrum obtained through linked scanning at constant $B / E$ on the hybrid tandem mass spectrometer following 8-kV collisions with helium in the first field-free region (CID spectrum not shown). It is worth noting that $\mathrm{m} / \mathrm{z} 138$ ions are also formed with high abundance by EI of solasodine [17]. High-energy product ions, which correspond to loss of $\mathrm{CH}_{4}$, of two molecules of $\mathrm{CH}_{4}$, and combined loss of $\mathrm{CH}_{4}$ and water, respectively, also are present at $m / z 400,384$, and 382. The elimination of $\mathrm{CH}_{4}$ can be rationalized as a charge-remote fragmentation that involves the angular methyl groups at $\mathrm{C} 10$ and/or C13, probably preceded by ring rupture.

Comparison of the high- and low-energy CID spectra reveals that the product ions at $m / z 114,126 / 124$, and 147 formed by low-energy processes are relatively more abundant in the high-energy CID spectrum than in the low-energy spectrum.

\section{Solasodine}

The low- and high-energy fragmentations observed in the CID spectrum of the $[\mathrm{M}+\mathrm{H}]^{+}$ions of solasodine (Figure $2 a$ and $b$, respectively) are summarized in Schemes IV and $V$, respectively. Ions $2 \mathrm{u}$ lower than ions in the CID spectra of tomatidine are present at $m / z 396,271,253$, and 159 in the low-energy spectrum. In addition, the low-energy CID spectrum shows relatively abundant ions in the low-mass region at $m / z 175,157,133,121,99$, and 70 . There are no ions at $m / z 161$, presumably because the double bond between $\mathrm{C} 5$ and $\mathrm{C} 6$ in the $\mathrm{B}$ ring of solasodine precludes their formation. The absence of these ions lends indirect support to the proposed fragmentation schemes (I and II) of the $m / z 161$ ions generated from the [M+ $\mathrm{H}]^{+}$ions of tomatidine.

High-energy product ions $2 \mathrm{u}$ lower than ions in the CID spectrum of the $[\mathrm{M}+\mathrm{H}]^{+}$ions of tomatidine are found at $m / z 398,380,342$, and 328. In the low-mass region, the product ions at $m / z 138$ (also seen in tomatidine) characteristic of high-energy CID and the product ions at $m / z 126 / 124$ formed at low collision energies are clearly discernible. Both the high- and low-energy CID spectra suggest that the origins of the
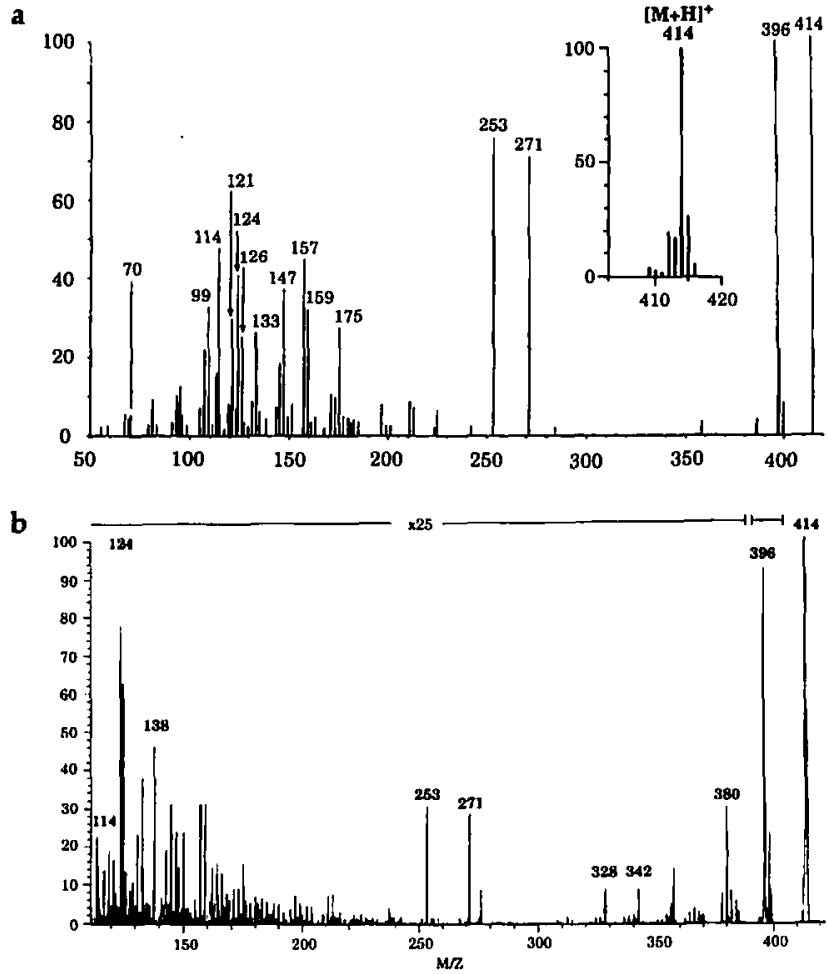

Figure 2. (a) Low-energy and (b) High-energy CID spectra of protonated molecules of solasodine. The molecule ion region of the (first-order) FAB spectrum (measured with the hybrid instrument) is shown in the insert of the low-energy CID spectrum (a).

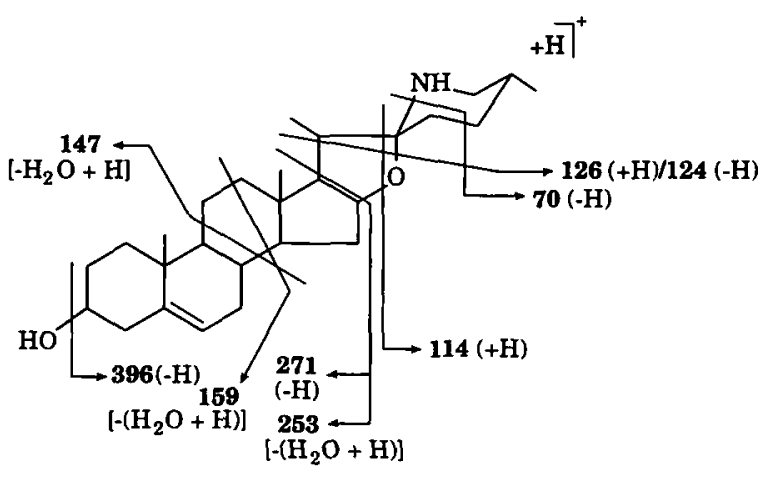

Scheme IV. Low-energy CID fragmentations of solasodine.

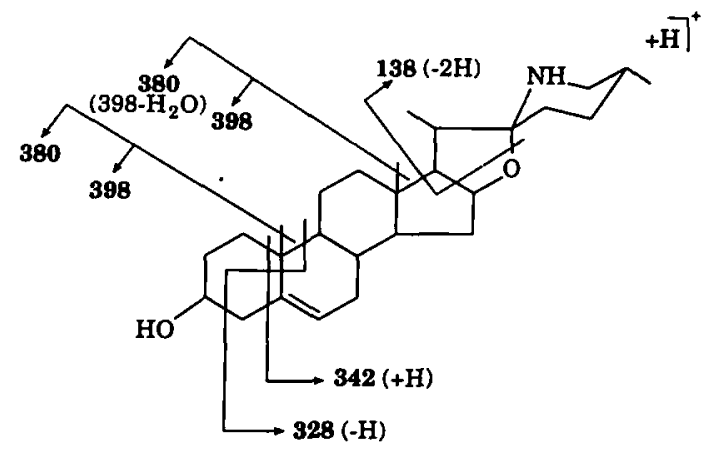

Scheme V. High-energy CID fragmentations of solasodine. 
ions in the low-mass region are quite complex and no attempt was made to explain all the ions in this region.

\section{Demissidine}

The low-energy fragmentations observed in the CID spectrum (Figure $3 \mathrm{a}$ ) of the $[\mathrm{M}+\mathrm{H}]^{+}$ions of demissidine are summarized in Scheme VI. A major fragmentation results in the formation of ions at $m / z 98$ that may be explained by multiple cleavages in the $E$ ring. The relative abundances of the ions at $m / z 150 / 151$ and at 204/205 were found to be strongly dependent on the collisional activation conditions: their intensities decreased significantly when the collision energy was
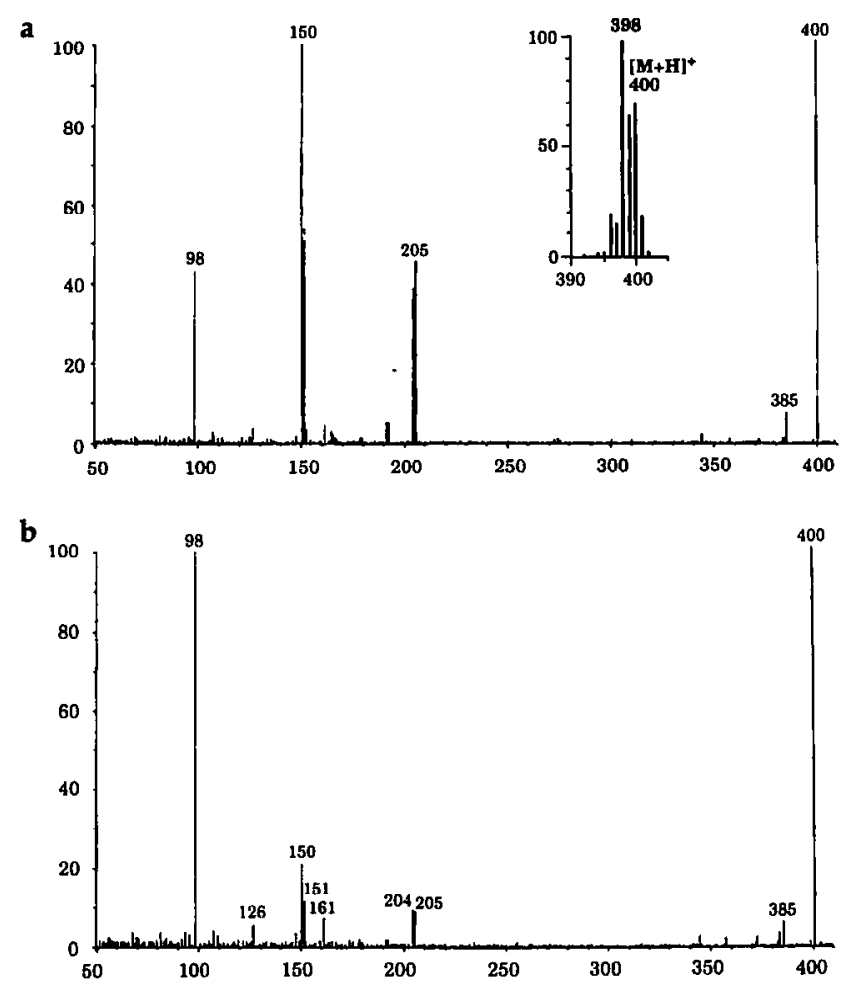

c

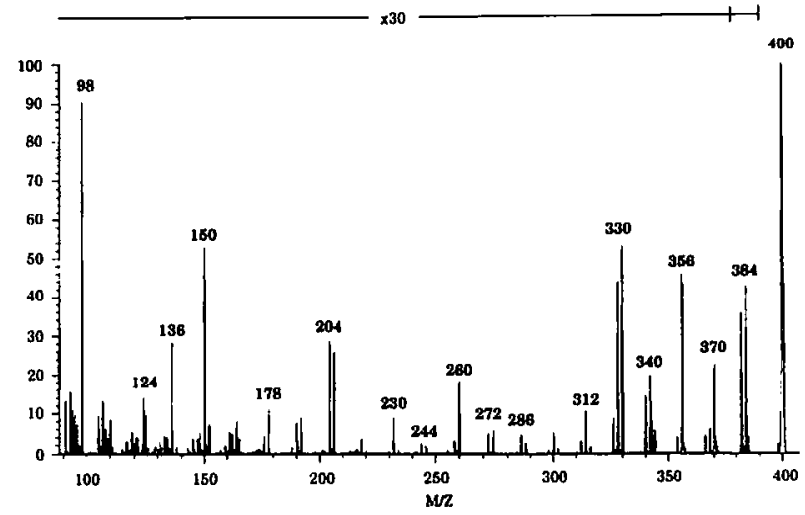

Figure 3. (a) $10 \mathrm{eV}$ and (b) $50 \mathrm{eV}$ low-energy and (c) highenergy CID spectra of protonated molecules of demissidine. The molecule ion region of the (first-order) FAB spectrum (measured with the hybrid instrument) is shown in the insert of the lowenergy CID spectrum (a).

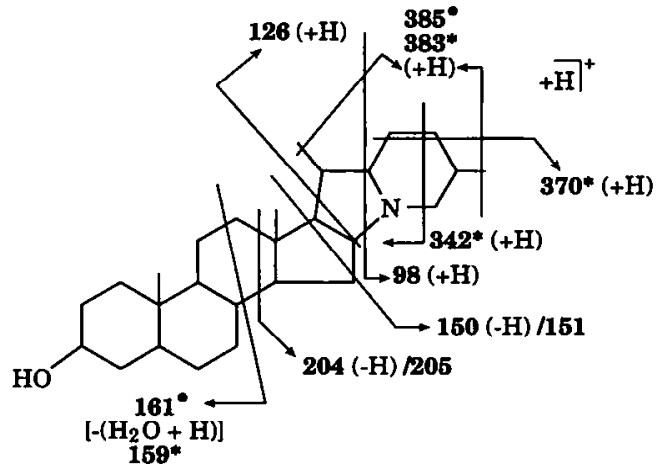

Scheme VI. Low-energy CID fragmentations of demissidine $(\bullet)$ and solanidine $(*)$ (where the structure of the latter incorporates a $\Delta^{5}$-double bond).

increased to $50 \mathrm{eV}$. Figure $3 \mathrm{~b}$ demonstrates the effects of increased collision energy on the low-energy CID spectrum of the $\left[\mathrm{M}+\mathrm{H}^{+}\right.$ions of demissidine. Comparison of Figures $3 \mathrm{a}$ and $\mathrm{b}$ shows that the ions at $\mathrm{m} / \mathrm{z}$ $150 / 151$ and $204 / 205$ are most abundant following very low-energy collisional activation $(10 \mathrm{eV}$ in the laboratory frame), conditions under which hydrogen rearrangements would be most likely to occur. The ions at $m / z \quad 150$ and 204 observed during high-energy CID (Figure 3c) are probably formed via a different fragmentation pathway. The formation of the ions at $\mathrm{m} / \mathrm{z} 150$ and 151 can be rationalized by multiple cleavages in the $\mathrm{D}$ ring. Following hydrogen rearrangement that induces cleavage of the $\mathrm{C} 15-\mathrm{C} 16$ bond, allylic cleavage of the $\mathrm{C} 13-\mathrm{C} 17$ bond may occur, which yields resonance-stabilized odd-electron ions at $\mathrm{m} / \mathrm{z}$ 151. It is significant that charge-remote allylic cleavages in unsaturated steroid rings of tauro-conjugated bile acid anions have been characterized as low-energy CID processes [13]. If allylic cleavages can occur remote from the charge, it is reasonable to assume that they also may occur proximate to the charge. The ions at $m / z 150$ may be rationalized by a double hydrogen rearrangement or by loss of $\mathrm{H}^{*}$ from the ions at $\mathrm{m} / \mathrm{z}$ 151. Although both mechanisms are illustrated in Scheme VII, the observation that the ion intensity ratio [ $m / z 150] /[m / z 151]$ was found to be independent of the collision energy can be interpreted as evidence against the ions at $m / z 151$ being precursors of the ions at $m / z 150$. It is notable that ions at $m / z 151$ were not observed in the high-energy CID spectrum. ${ }^{13} \mathrm{C}$ contributions from $\mathrm{M}^{+\cdot}$ ions that are formed in the 3-nitrobenzyl alcohol matrix and are sampled together with $[\mathrm{M}+\mathrm{H}]^{+}$ions cannot be excluded because of the significant relative abundance of the $\mathrm{M}^{+\cdot}$ ions (ion intensity ratio $\left.\left[\mathrm{M}^{+\cdot}\right] /[\mathrm{M}+\mathrm{H}]^{+}=9 / 10\right)$. However, in that case it is hard to explain the effect of the collision energy, more specifically, the absence of the ions at $m / z 151$ and 205 (vide infra) at high-energy CID. The common mass difference of $1 \mathrm{u}$ for the ions at $\mathrm{m} / \mathrm{z}$ $150 / 151$ and 204/205 might suggest similar fragmentation pathways. Plausible mechanisms, which involve 


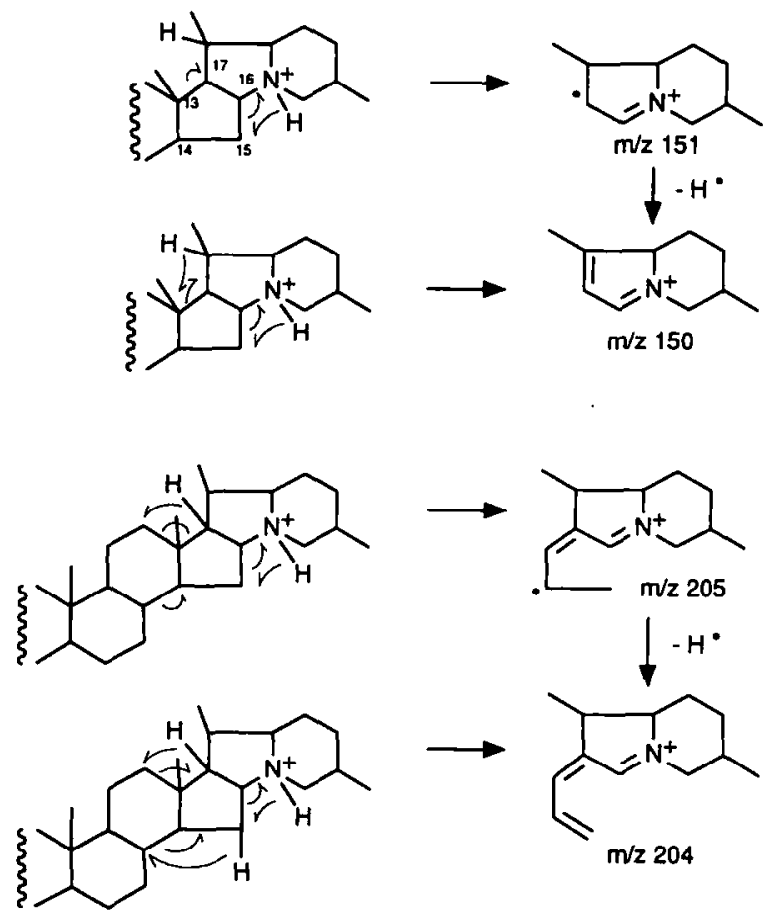

Scheme VII. Mechanisms that rationalize the formation of $m / z$ $150 / 151$ and $204 / 205$ in the low-energy CID spectrum of demissidine.

hydrogen rearrangement(s) and multiple cleavages in the $C$ and D rings, are proposed in Scheme VII. It is again noteworthy that the odd-electron ions at $m / z$ 205 are absent from the high-energy CID spectrum. Ions at $m / z 150$ and 204 have been reported also to occur with high abundance in the EI spectrum of solanidine [17], which only differs from demissidine by the presence of a C5-C6 double bond in the former, and in the EI spectra of solanidine $\mathrm{N}$-oxides [18]. The formation of the low-energy CID product ions at $\mathrm{m} / \mathrm{z}$ 126 and 161, present at low abundances following higher-energy ( $50 \mathrm{eV}$ laboratory frame) collisional activation, are also rationalized in Scheme VI. The minor low-energy CID product ions in the upper mass region at $m / z 385$ and 383 correspond to the loss of $\mathrm{CH}_{3}$ and the combined loss of $\mathrm{CH}_{3}^{-}$and $\mathrm{H}_{2}$, respectively, and may be interpreted by charge-proximate fragmentation in the $\mathrm{N}$-containing $\mathrm{E} / \mathrm{F}$-ring system. Loss of $\mathrm{CH}_{3}$ from the $\mathrm{M}^{+\cdot}$ ions has been observed in the EI spectrum of solanidine [17]. Here again, ${ }^{13} \mathrm{C}$ contributions from $\mathrm{M}^{+\cdot}$ ions which are sampled together with $[M+$ $\mathrm{H}^{+}$ions cannot be excluded. With respect to the loss of $\mathrm{CH}_{3}$ radicals from $[\mathrm{M}+\mathrm{H}]^{+}$ions, it is worth mention that this unusual transition from even- to odd-electron ions has been reported for methylated flavonoid aglycons [19].

Fragmentations in the high-energy CID spectrum of the $[\mathrm{M}+\mathrm{H}]^{+}$ions of demissidine (Figure $3 \mathrm{c}$ ) are rationalized in Scheme VIII. Most of these fragmentations can be interpreted as arising through charge-remote reactions that involve multiple cleavages in the $A, B$,

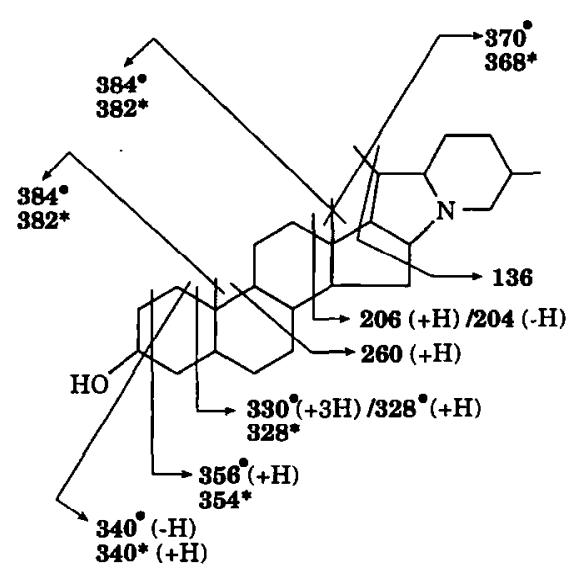

$+\mathrm{H}^{+}$

Scheme VIII. High-energy CID fragmentations of demissidine $(\bullet)$ and solanidine $(*)$ (where the structure of the latter incorporates a $\Delta^{5}$-double bond).

or $\mathrm{C}$ rings, together with $\mathrm{CH}_{4}$ loss, which most probably involves the angular methyl groups. The ions at $m / z 136$ may be explained by a charge-proximate fragmentation with multiple cleavages in the $\mathrm{D}$ ring, loss of $\mathrm{CH}_{3}$ from $\mathrm{C} 18$ and further elimination of $\mathrm{H}$ : The ions at $m / z 370$ correspond to the combined loss of two $\mathrm{CH}_{3}$, possibly the methyl groups at $\mathrm{C} 13$ and C18.

\section{Solanidine}

Fragmentations observed in the low-energy CID spectrum of the $[\mathrm{M}+\mathrm{H}]^{+}$ions of solanidine (Figure $4 \mathrm{a}$ ) appear to be essentially similar to those found for demissidine (Schemes VI and VII). An increase in the collision energy from 10 to $50 \mathrm{eV}$ (Figure $4 \mathrm{~b}$ ) resulted in a significant reduction in the abundances of the ions at $m / z \quad 150 / 151$ and $204 / 205$ and the appearance of ions with low abundance at $m / z 126,342,370,381$, and 383. The ions at $m / z 383$ and 381 correspond to the loss of $\mathrm{CH}_{3}$ and the combined loss of $\mathrm{CH}_{3}^{-}$and $\mathrm{H}_{2}$, respectively, and parallel similar fragmentations of the $[\mathrm{M}+\mathrm{H}]^{+}$ions of demissidine, which yield the ions at $m / z 385$ and 383 (see above). The ions at $m / z 370$ and 342 are consistent with the loss of $\mathrm{C}_{2} \mathrm{H}_{4}$ and $\mathrm{C}_{4} \mathrm{H}_{8}$, respectively, and may be interpreted by invoking fragmentation in the N-containing F-ring system, as described in Scheme VI.

The high-energy CID spectrum of the $[\mathrm{M}+\mathrm{H}]^{+}$ ions of solanidine (Figure 4c) is also rationalized in Scheme VIII and contains fragmentation processes analogous to those of demissidine.

\section{$\alpha$-Tomatine}

Glycosidic and glycoside-aglycon cleavages discussed in the following text are assigned by using the nomenclature of Domon and Costello [20]. Fragmentations observed in the low-energy CID spectrum (Figure 5a) of the $[\mathrm{M}+\mathrm{H}]^{+}$ions of $\alpha$-tomatine are summarized 


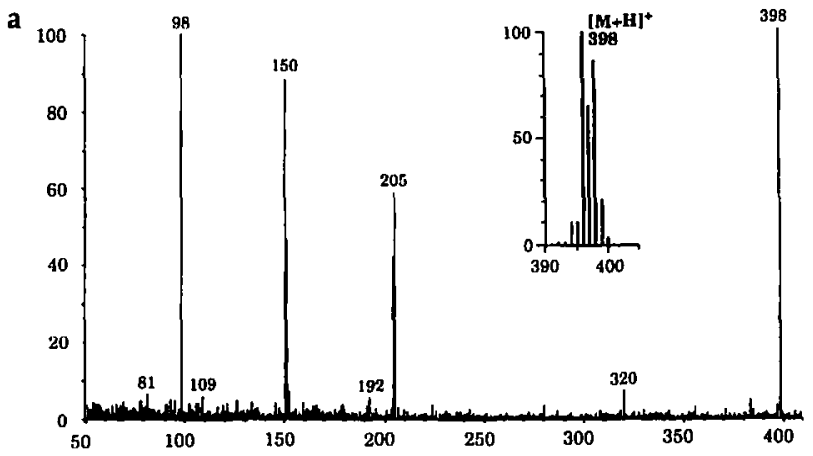

b

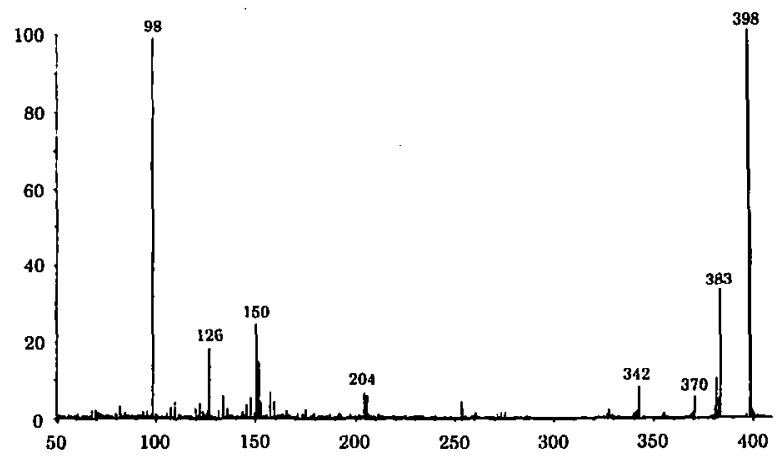

c

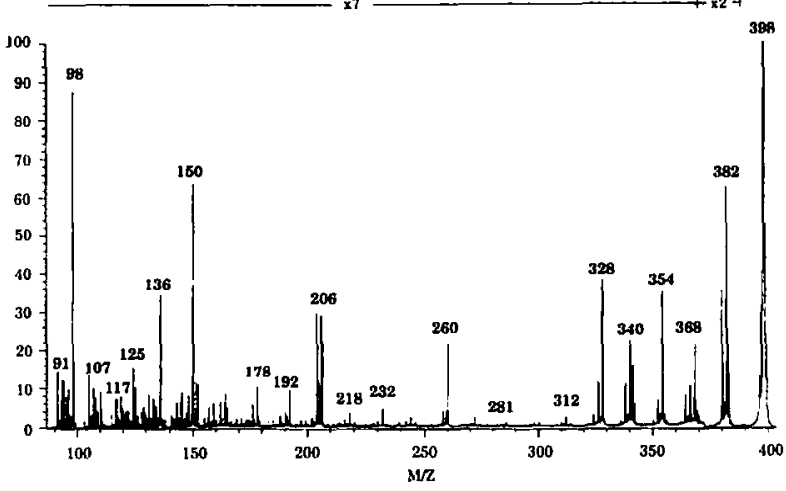

Figure 4. (a) $10 \mathrm{eV}$ and (b) $50 \mathrm{eV}$ low-energy and (c) highenergy CID spectra of prolonated molecules of solanidine. The molecule ion region of the (first-order) FAB spectrum (measured with the hybrid instrument) is shown in the insert of the lowenergy CID spectrum (a).

in Scheme IX. A major fragmentation is the loss of water to give ions at $m / z$ 1016. This fragmentation probably arises through a rearrangement process in ring $\mathrm{E}$, rather than by decomposition of the carbohydrate moiety, because loss of water is not observed in the low-energy CID spectra of the $[\mathrm{M}+\mathrm{H}]^{+}$ions of $\alpha$-chaconine and $\alpha$-solanine. Ions typical of the aglycon moiety (already discussed) are present at $\mathrm{m} / \mathrm{z} 416$ $\left(Y_{0}^{+}\right), 398,273$ and 255 . The ions at $m / z 380$ correspond to $\left[\mathrm{Y}_{0}^{+}-\left(2 \times \mathrm{H}_{2} \mathrm{O}\right)\right]$ and may be formed from the $\left[\mathrm{M}+\mathrm{H}-\mathrm{H}_{2} \mathrm{O}\right]^{+}$ions $(m / z$ 1016). Ions also are present in the low-mass region at $m / z 163,145,133$, $126 / 124$, and 115 . The ions at $\mathrm{m} / z 163$ and 133 correspond to $\mathrm{B}_{1}^{+}$ions and are characteristic of terminal hexose and pentose residues, respectively. The ions at $m / z 145$ and 115 may arise by loss of water from the

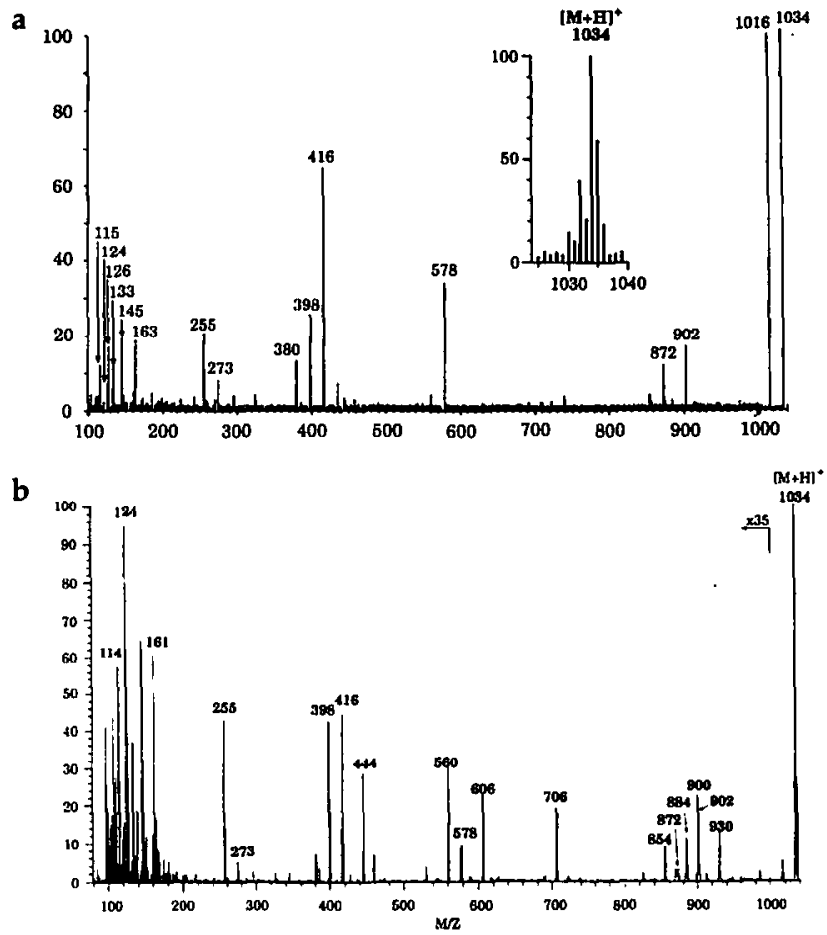

Figure 5. (a) Low-energy and (b) high-energy CID spectra of protonated molecules of $\alpha$-tomatine. The molecule ion region of the (first-order) FAB spectrum (measured with the hybrid instrument) is shown in the insert of the low-energy CID spectrum (a).

ions at $m / z 163$ and 133, respectively. The ions at $m / z$ $126 / 124$ are characteristic of the aglycon moicty and already have been rationalized in Scheme I. The lowenergy CID spectrum shows a clear series of $Y_{n}^{+}$ions at $m / z 902\left(Y_{2 \beta}^{+}\right), 872\left(Y_{2 \alpha}^{+}\right), 578\left(Y_{1}^{+}\right)$, and $416\left(Y_{0}^{+}\right)$, which are fully consistent with the carbohydrate sequence.

Loss of water is not a major fragmentation in the corresponding high-energy CID spectrum (Figure 5b), which contains, in addition to the ions already described in the low-energy spectrum, prominent fragment ions (Scheme $X)$ at $\mathrm{m} / \mathrm{z} 930\left({ }^{1,5} \mathrm{X}_{2 \alpha}\right), 900\left({ }^{1,5} \mathrm{X}_{2 \alpha}\right)$, $884\left(Z_{2 \beta}\right), 854\left(Z_{2 \alpha}\right), 706\left(Z_{2 \alpha / 2 \beta}+2 H\right), 606\left({ }^{1,5} X_{1}\right)$, $560\left(Z_{1}\right), 444\left({ }^{1,5} X_{0}\right)$, and 161 (probable aglycon fragment). These ions indicate that fragmentation of the sugar rings and of the interglycosidic bonds proximal to the aglycon moiety are high-energy processes.

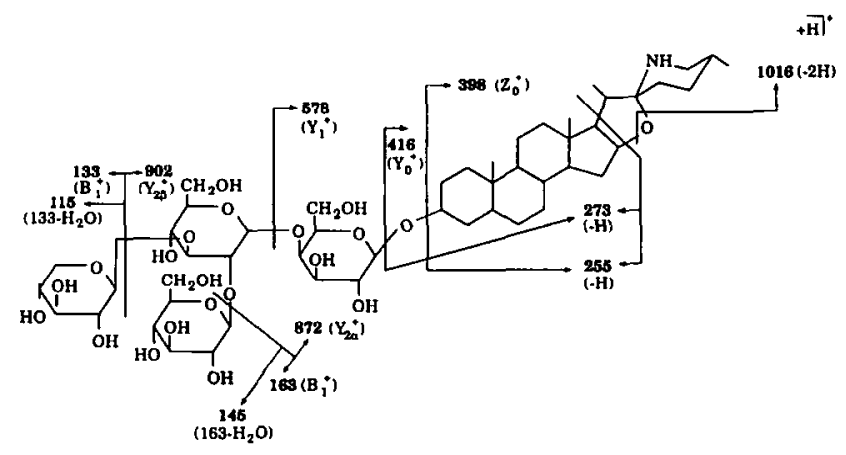

Scheme IX. Low-energy CID fragmentations of $\alpha$-tomatine. 


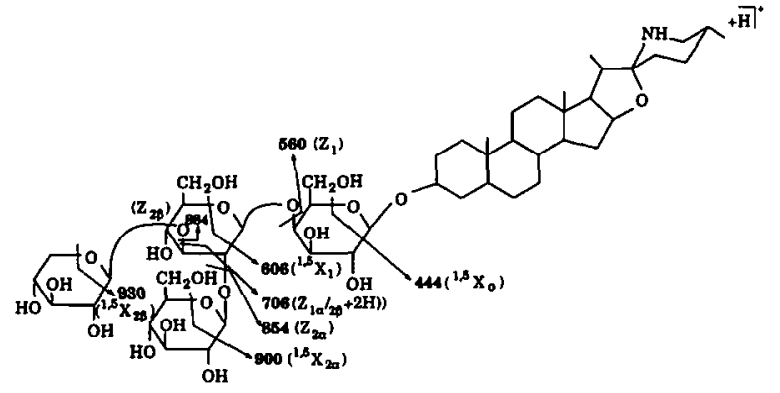

Scheme X. High-energy CID fragmentations of $\alpha$-tomatine.

\section{$\alpha$-Chaconine and $\alpha$-Solanine}

These two glycoalkaloids have similar structures and fragmentation patterns and are discussed together. Fragmentations observed in the low-energy CID spectrum of $\alpha$-solanine (Figure 6a) are rationalized in Scheme XI. Ions present at $m / z 837$ and 853 in the low-energy CID spectrum of the $[\mathrm{M}+\mathrm{H}]^{+}$ions of $\alpha$-chaconine (Figure 7a) and $\alpha$-solanine, respectively, are formed by the loss of $\mathrm{CH}_{3}$, a fragmentation characteristic of the aglycon moiety. The lower-mass regions exhibit typical distinct aglycon ions at $m / z 98$, 150, and 204. The ions at $m / z 150$ and 204 may be rationalized by the double hydrogen rearrangement process described above (Scheme VII). The low-energy CID spectrum of the $[\mathrm{M}+\mathrm{H}]^{+}$ions of $\alpha$-chaconine also exhibits sequence-related $\mathrm{Y}_{n}^{+}$ions at $m / z 706$

a

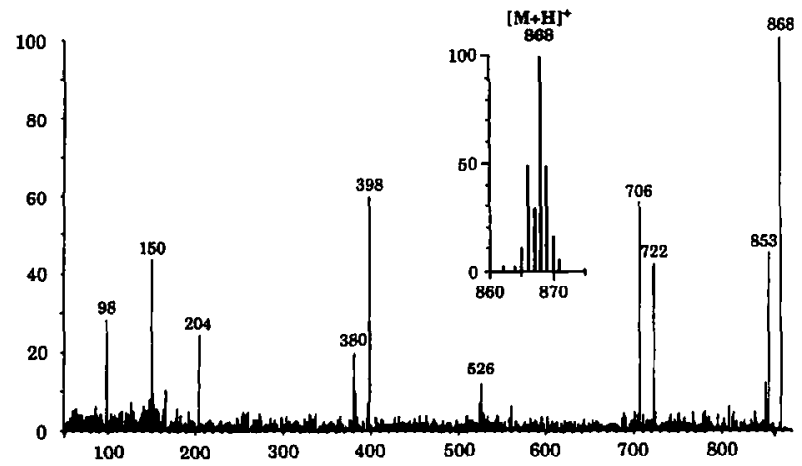

b

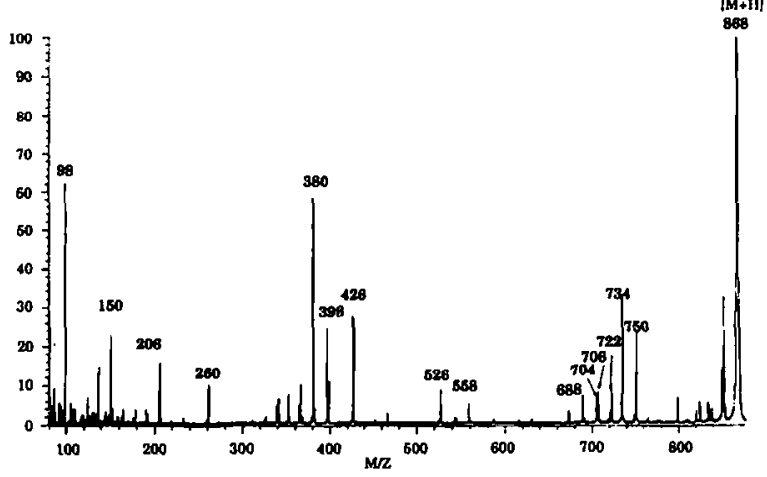

Figure 6. (a) Low-energy and (b) high-energy CID spectra of protonated molecules of $\alpha$-solanine. The molecule ion region of the (first-order) FAB spectrum (measured with the hybrid instrument) is shown in the insert of the low-energy CID spectrum (a).

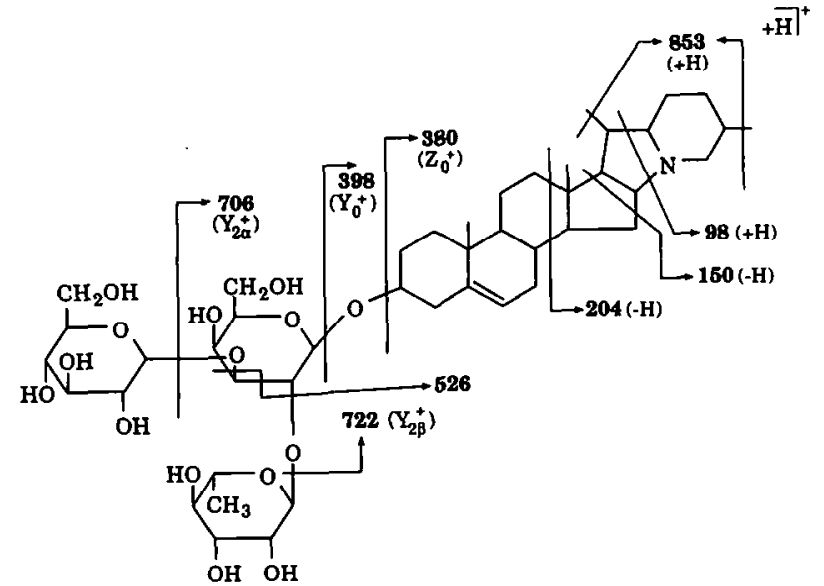

Scheme XI. Low-energy CID fragmentations of $\alpha$-solanine.

$\left(\mathrm{Y}_{1}^{+}\right)$and $398\left(\mathrm{Y}_{0}^{+}\right)$, as well as low-abundance $\mathrm{Z}_{\mathrm{n}}^{+}$ions at $m / z 688\left(\mathrm{Z}_{1}^{+}\right)$and $380\left(\mathrm{Z}_{0}^{+}\right)$. CID of the $[\mathrm{M}+\mathrm{H}]^{+}$ ions of $\alpha$-solanine generates $\mathrm{Y}_{1}^{+}$ions at $m / z 722\left(\mathrm{Y}_{1 \beta}^{+}\right)$ and $706\left(Y_{1 \alpha}^{+}\right)$, which correspond to the loss of terminal rhamnose and galactose residues, respectively. Ions observed at $\mathrm{m} / z 526$ are formed by the loss of the two terminal sugar residues (Scheme XI).

The high-energy CID spectra of the $[\mathrm{M}+\mathrm{H}]^{+}$ions of $\alpha$-solanine (Figure $6 \mathrm{~b}$ ) exhibit prominent ions resulting from fragmentation processes (Scheme XII) at $\mathrm{m} / \mathrm{z}$ $750\left({ }^{1,5} X_{1 \beta}\right), 734\left({ }^{1,5} X_{1 \alpha}\right), 704\left(Z_{1 \beta}\right), 688\left(Z_{1 \alpha}\right)$, and 426 $\left({ }^{1,5} X_{0}\right), \alpha$-Chaconine (Figure $7 \mathrm{~b}$ ) exhibits ions formed
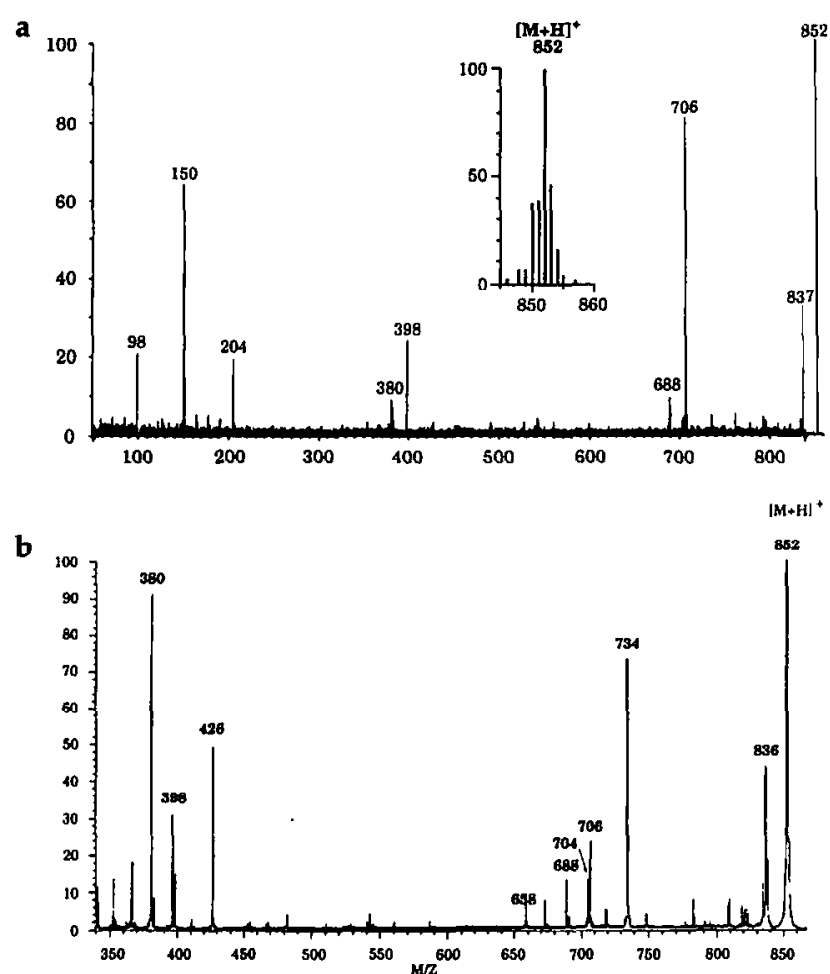

Figure 7. (a) Low-energy and (b) high-energy CID spectra of protonated molecules of $\alpha$-chaconine. The molecule ion region of the (first-order) FAB spectrum (measured with the hybrid instrument) is shown in the insert of the low-energy CID spectrum (a). 


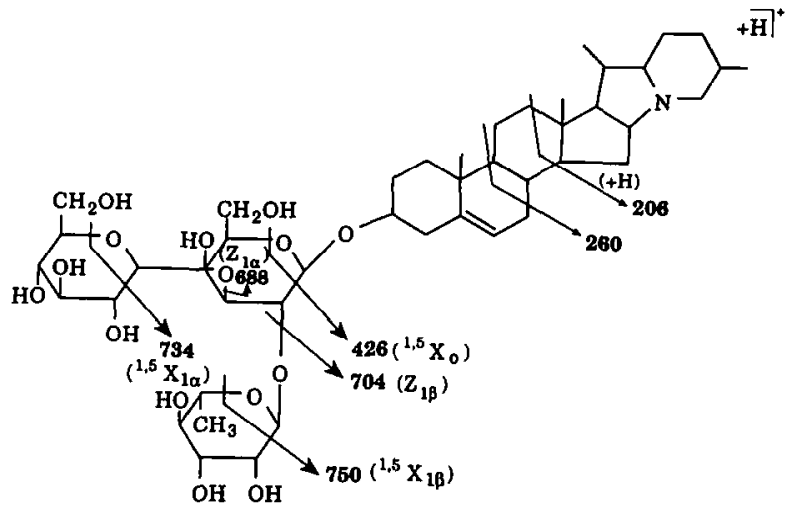

Scheme XII. High-energy CID fragmentations of $\alpha$-solanine.

by high-energy processes at $\mathrm{m} / \mathrm{z} 734\left({ }^{1,5} \mathrm{X}_{1 \alpha}\right.$ and $\left.{ }^{1,5} X_{1 \beta}\right), 704\left({ }^{1,5} X_{1 \alpha}-\mathrm{HCHO}\right.$ and ${ }^{1,5} X_{1 \beta}$-HCHO), and 426 $\left({ }^{1,5} X_{0}\right)$, in addition to those ions previously discussed in the low-energy spectrum. The additional ions add support to the hypothesis (see the preceding discussion of $\alpha$-tomatine) that cleavage across the sugar rings is a high-energy process. Cleavage at the interglycosidic bonds proximal to the aglycon is again more prominent in the high-energy spectrum.

\section{Conclusions}

The low- and high-energy CID spectra of $[\mathrm{M}+\mathrm{H}]^{+}$ ions of glycoalkaloids and aglycons contain a wealth of information, which includes the mass of the aglycons, the carbohydrate sequence and linkage sites, and ions characteristic of different structural features in the aglycon moieties. Low-energy CID favors chargedriven fragmentation of the aglycon rings, whilst high-energy CID spectra are more complex and contain additional ions that appear to result from chargeremote fragmentations, multiple cleavages, or complex charge-driven rearrangements. Low-energy CID fragmentations of sugar residues in the ylycoalkaloids generate $\mathrm{Y}_{n}^{+}$ions and some low intensity $Z_{n}^{+}$ions; the high-energy spectra also exhibit strong ${ }^{1.5} X_{n}^{+}$ions, formed by multiple cleavage of the sugar ring, and significant $\mathrm{Z}_{n}^{+}$ions. These data indicate that chargeremote multiple cleavage processes are promoted by high-energy CID. The $Z_{n}^{+}$ions, which are also formed by low-energy CID and which appear to be formed by a simple cleavage reaction accompanied by a single $\mathrm{H}$ loss, may result from a rearrangement process, as has been demonstrated in the case of $Z_{\|}^{+}$ions formed upon low-energy CID of protonated flavonoid $\mathrm{O}$-glycosides [21].

It should be pointed out that the mass spectrometric methods described in this study alone are not sufficient to elucidate the complete chemical structures of unknown glycoalkaloids; more specifically, NMR spectroscopic and chemical methods are required to determine specific carbohydrate residues, substitution positions on both carbohydrates and aglycon, and structural details of the aglycon. However, the integra- tion of modern tandem mass spectrometric techniques with advanced NMR techniques is of significant power and can greatly facilitate the structure elucidation of complex glycoconjugates such as glycoalkaloids and saponins. Structure elucidation by NMR techniques requires sample amounts that are at least a hundredfold higher than for tandem mass spectrometry; thus, in cases where the sample is limited, tandem mass spectrometry, especially in combination with sensitive photodiode array detection, is indicated to obtain partial chemical structure information.

\section{Acknowledgments}

We are grateful to the Belgian Nationaal Fonds voor Wetenschappelijk Onderzoek (NFWO) and the British Biotechnology and Biological Sciences Research Council (BBSRC) for financial support. M. Claeys is indebted to the NFWO as a research director.

\section{References}

1. Facino, R. M.; Carini, M.; Traldi, P.; Belli, B.; Gioia, B.; Arlandini, E. Biomed. Eniron. Mnss Spectrom. 1987, 14, 187-194.

2. Chen, Y.; Chen, N.; Li, H.; Zhao, F.; Chen, N. Biomed. Environ. Mass Spectrom. 1987, 14, 9-15.

3. Tomer, K. B.; Gross, M. L. Bioned. Environ. Mass Spectrom. $1988,15,89-98$

4. Evans, S.; Buchanan, R.; Hoffman, A.; Mellon, F. A.; Price, K. R.; Walls, F. C.; Hall, S.; Burlingame, A. L.; Chen, S.; Derrick, P. J. Org. Mass Spectrom. 1993, 28, 289-290.

5. Chen, S.; Derrick, P. J.; Mellon, F. A.; Price, K. R. Anal. Biochem. 1994, 218, 157-169.

6. Liehr, J. G.; Kingston; E. E.; Beynon, J. H. Biomed. Mass Spectrom. 1985, 12, 95-99.

7. Tomer, K. B.; Gross, M. L. Biomed. Enoiron. Mass Spectrom. 1986, 15, 89-98.

8. Griffiths, W. J.; Zhang, J.; Sjövall, J. Rapid Commun. Mass Spectrom. 1993, 7, 235-240.

9. Griffiths, W. J.; Zhang, J.; Sjövall, J. Rapid Commun. Mass Spectrom. 1994, 8, 227-236.

10. Libert, R.; Hermans, D.; Draye, J.-P.; Van Hoof, F.; Sokal, E.; de Hoffmann, E. Clin. Chem. 1991, 37, 2102-2110.

11. Eckers, C.; East, P. B.; Haskins, N. J. Biol. Mnss Spectrom. 1991, 20, 731-739.

12. Wood, K. V.; Sun, Y.; Elkin, R. G. Anal. Chem. 1991, 63, 247-250.

13. Stroobant, V.; Libert, R.; Van Hoof, F.; de Hoffmann, E. J. Am. Soc. Mass Spectrom. 1995, 6, 588-596.

14. Rossi, S.-A.; Johnson, J. V.; Yost, R. A. Biol. Mass Spectrom. 1994, 23, 131-139.

15. Marschall, H.-U.; Griffiths, W. J.; Zhang, J.; Wietholtz, H.; Matern, H.; Matern, S.; Sjövall, J. I. Lipid Res. 1994, 35, 1599-1610.

16. Coxon, D. T.; Price, K. R.; Jones, R. D. J. Sci. Food Agric. 1979, 30, 1043-1047.

17. Budzikiewiecz, H. Tet rahedron 1964, 20, 2267-2278.

18. Quyen, L. T.; Schmidt, J.; Schreiber, K. I. Mass Spectrom. 1995, 30, 201-205.

19. Li, Q. M.; Van den Heuvel, H.; Claeys, M. Procuedings of the 43rd Annual ASMS Conference on Mass Spectrometry and Allied Topics, Atlanta, GA, 1995, p. 1181.

20. Domon, B.; Costello, C. E. Glycoconjugate J. 1988, 5, 397-409.

21. Li, Q. M.; Claeys, M. Biol. Mass Spectrom. 1994, 23, 406-416. 